\title{
Improving the Mechanical and Thermal Properties of Shish-kebab via Partial Melting and Re-crystallization
}

\author{
Dashan Mi ${ }^{1,2}$, Jie Zhang ${ }^{2}$, Kim Ragaert ${ }^{1}$
}

1. Ghent University, Gent, Belgium

2. Sichuan University, Chengdu, China

\begin{abstract}
A fully oriented isotactic polypropylene (iPP) sample containing a large amount of shish-kebab was molded by multiflow vibrate-injection molding. When annealing was performed at a temperature close to the melting temperature of the shish-kebab, thin-kebab can be melted and release chains to the amorphous phase. The released oriented chains can reduce the concentration of the molecule entanglements and facilitate the molecular chains to rearrange on the thick-lamella.In situ small-angle X-ray scattering and wide-angle Xray diffraction were performed to elucidate the melt-recrystallization process. Results showed that annealing at $165^{\circ} \mathrm{C}$ can melt thin kebab but thicken the thick kebab, and the kebab grew in only one dimension. We focused on the complex melting-recrystallization processes and provided a theoretical basis for the highefficient, time-saving annealing method. (This work has already been published in European Polymer Journal 101 (2018): 1-11.)
\end{abstract}

\section{INSTRUCTIONS}

In most polymer processing methods, the physical properties and crystallization of polymer melts undergoing a complex flow field are considerably affected. Thermal annealing is one of the common post treatments for improving semicrystalline polymer properties. Annealing between the glass transition temperature $(\mathrm{Tg})$ and melting temperature $(\mathrm{Tm})$ can effectively improve the microstructures and mechanical properties of polymers [Rastogi et al. 1997, Yao et al. 2008]. Under annealing, an oriented structure differs significantly from an isotropic sample regarding crystallinity; because aligned materials readily crystallizes as the chains are already elongated. For an oriented structure (e.g., shish-kebabs), the oriented shish can provide nucleating sites for the lateral growth of new kebabs. As far as we know, the partial melting and recrystallization process of shish-kebab have not been revealed before.

Using a temperature near the melting temperature can greatly reduce the annealing time. However, the process of melting and recrystallization in this temperature is complex, and high temperatures can sometimes lead to a dramatic decrease in the overall crystalline fraction. Given that melting temperatures differ from the variation in crystalline modifications, polymorphism is the primary reason for the complex melting and recrystallization behavior of crystalline polymers. Additionally, even the same phase can exhibit a complex melting temperature. Finally, the melting of metastable crystals often facilitates a phase transformation that produces stable crystals. This behavior indicates that the melting and recrystallization behaviors are complex owing to morphology reorganization, which extends the long period and increases the thicknesses of lamellae. Two major mechanisms can explain lamellar thickening: one is the sliding diffusion mechanism in which the polymer chains in the lamellar crystal slide through the crystal lattice along the chain axis, [Bai et al. 2012] and the other is the melting-recrystallization mechanism [Mandelkern et al. 2006]. Some researchers found that the crystallinity of polyethylene initially decreases and then increases during the annealing process during recrystallization; they also suggested that the annealing process is the partial melting of the crystalline, followed by their recrystallization.

In a previous article, we used the multiflow vibrate-injection molding (MFVIM) to form a fully oriented isotactic polypropylene (iPP) injectionmolded sample, which has a shish-kebab content ratio higher than 95\% [Mi et al. 2016]. This fully oriented sample enabled us to study the effect of the melting-recrystallization mechanism on shishkebab. In this study, we used a high temperature (close to Tm) for the first time when we annealed a highly oriented injection-molded part. The impact strength and the melting temperature dramatically increased by approximately $158 \%$ and $10{ }^{\circ} \mathrm{C}$, respectively. In situ melting can improve our understanding of complex annealing processes. Thinkebab is melted and release chains to the amorphous 
phase. Then these chains rearrange on the thick lamella and the lamella grew in the flow direction. In the present study, we aim to demonstrate the complex melting-recrystallization process.

\section{EXPERIMENTAL SECTION}

iPP (trade name T30S) was purchased from Dushanzi Petroleum Chemical Co., China. The fully oriented sample was prepared. In situ X-ray characterization was used. SAXS and WAXD were performed during the same session, although not simultaneously, at the BL16B1 beam line in the Shanghai Synchrotron Radiation Facility (SSRF), Shanghai, China. The orientation of lamellar crystals in the injection-molded parts was calculated.

\section{RESULTS AND DISCUSSION}

Fully oriented iPP samples were tested via in situ SAXS and WAXD to elucidate the meltingrecrystallization process. The temperature protocol used during the experiments is shown in Figure 1 (a). The shish-kebab was heated to $165{ }^{\circ} \mathrm{C}$ with a heating rate of $10{ }^{\circ} \mathrm{C} / \mathrm{min}$ and held at this temperature for $40 \mathrm{~min}$. The sample was then heated to 200 ${ }^{\circ} \mathrm{C}$ at the same heating rate. Figures 1 (b), (c), (d), and (e) focuses on the initial stages of annealing (green area in Figure 1 [a]). Figure 1 (b) shows that when temperature increased from $100{ }^{\circ} \mathrm{C}$ to $165^{\circ} \mathrm{C}$, the intensity profiles of the fan-shaped integrated $2 \mathrm{D}$ SAXS patterns in the meridional direction shifted from right to left, and the scattering intensity continues to rise at increasing annealing time. Figure 1 (c) shows that the integrated 2D SAXS patterns are in an equatorial direction. The scattering intensity also increased along with temperature increase. This scattering intensity was elicited by the shish or microfibrils and a *-axis-oriented "daughter" lamellae as mentioned before. Therefore, the growth or improvement of the shish structure cannot be contributed to increased intensity. Figure 1 (d) presents the azimuthal scan of the (040) lattice plane. At increasing temperature, the height of the peak declined and then increased. Figure 9 (e) shows the one-dimensional (1D)-WAXD curves and absence of significant changes.

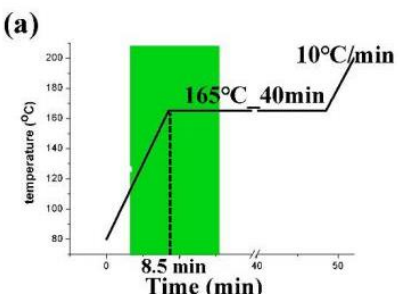

(b)
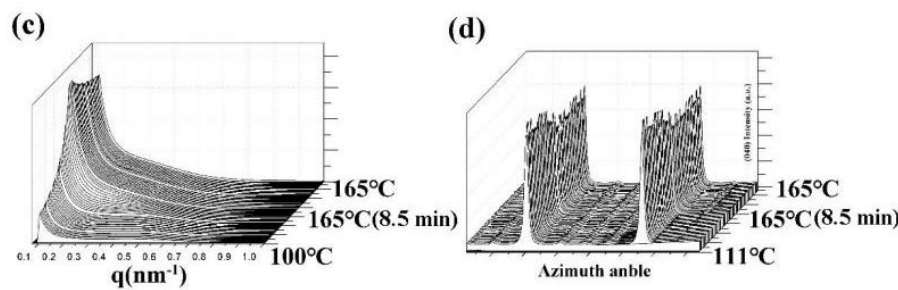

(e)

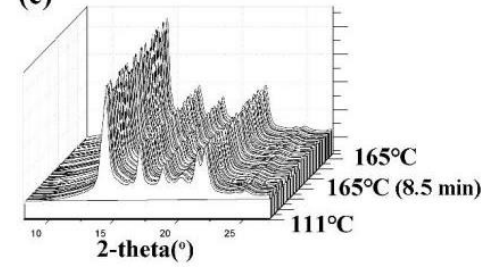

Figure 1. (a) Temperature and time protocol for the in suit WAXS/SAXS experiments, the intensity profiles of fan-shaped integrated 2D SAXS patterns in (b) meridional and (c) equatorial directions, (d) Azimuthal scan of (040) lattice plane, (e) plots of integrated WAXD intensity as a function of $2 \theta$. [Mi et al. 2018]

The degree of orientation, long period, crystallinity (Xc), and lamella thickness were calculated from Figure 9 and shown in Figure 1. The molecular orientation was enhanced by annealing, as shown in Figure 2. This phenomenon was also observed in the melt-spun iPP monofilaments that recrystallized during annealing. The local reorientation of the amorphous strands between adjacent lamellae enhanced the degree of orientation [Li et al. 2012]. In our case, not only crystallization contributed to the orientation but also melting. When the thin lamella melted, the average thickness increased, because the degree of orientation of a thin lamella was lower than that of a thick lamella in most cases. When the temperature reached $165{ }^{\circ} \mathrm{C}$, the degree of orientation declined, which also indicated that some oriented lamellae melted. In Figure 2 (b), the period continued to increase when the temperature increased from $132{ }^{\circ} \mathrm{C}$ to $165^{\circ} \mathrm{C}$. By contrast, after $500 \mathrm{~s}$, L remained stable at $19.5 \mathrm{~nm}$. The increased $\mathrm{L}$ value was attributed to the falling crystallinity, as shown in Figure 2 (c). The lamellar thickness had an upward trend, which was contributed by decreasing thin lamella and increasing thick lamella (Figure 2 [d]). 
(a)

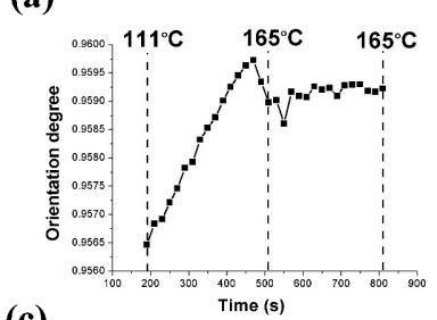

(c)

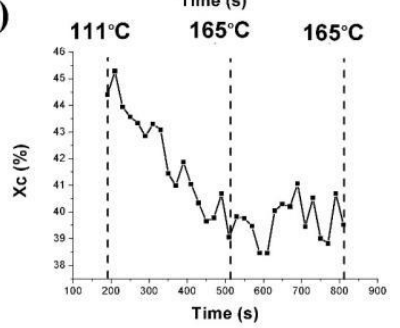

(b)

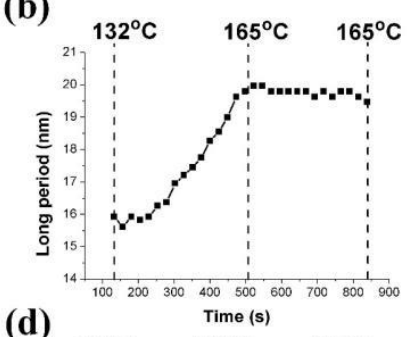

(d)

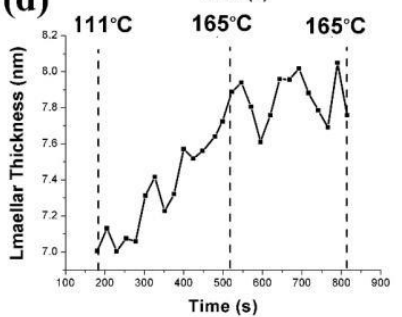

Figure 2. Various results as a function of annealing duration (s) (a) Orientation degree, (b) Long period, (c) crystallinity, (d) lamellar thickness. [Mi et al. 2018]

To clarify the relative speed of melting and recrystallization, as well as the melting and growth kinetics. The evolution of space filling overtime for the experiments is calculated using Equation (1).

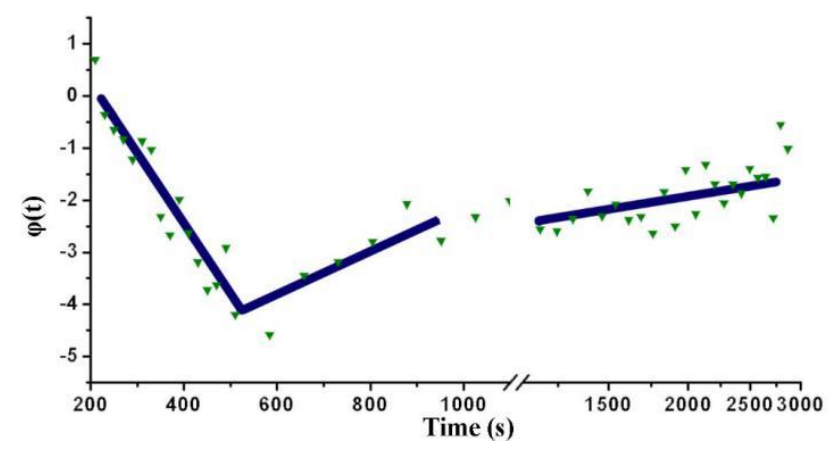

Figure 3. The value of $\varphi(t)$ is represented by an inverted triangle, and these values are fitted by blue lines (Log 10 scale after break). [Mi et al. 2018]

$$
\varphi(t)=\frac{x(t)-x_{0}}{x_{\infty}-x_{0}}
$$

where $\mathrm{x}(\mathrm{t})$ is the crystallinity index, and $\mathrm{x} 0$ and $\mathrm{x} \infty$ are the initial and final crystallinity, respectively. Then, the value of $\varphi(t)$ is represented by an inverted triangle as shown in Figure 3. The line, which fitted real dates well, indicating that crystal volume of increased or decreased in a straight line. It is noteworthy that when the temperature is stabilized at $165^{\circ} \mathrm{C}$ (after 500s), $\varphi(\mathrm{t})$ will rise rapidly in the following 500s (500s-1000s). After that, $\varphi(t)$ will rise slowly from 1000s to 3000s. For the crystallization process, the straight line means that the kebab only grows in one direction exhibiting as one-dimensional growth.

\section{CONCLUSIONS}

Using in situ WAXD/SAXS, we investigated the melting-recrystallization process and found that the shish-kebab structure can be refined at a temperature as high as $165{ }^{\circ} \mathrm{C}$. For a two mm-thick sample, the melting-recrystallization process mainly occurred in the first $500 \mathrm{~s}$. The unstable lamella can be removed, and the thickness of the thick lamella can be increased. During annealing, the total crystallinity initially decreased and then increased, and lamella grew in only one direction.

\section{PREFERENCES}

- Rastogi S, Spoelstra A, Goossens J, Lemstra P. Chain mobility in polymer systems: on the borderline between solid and melt. 1. Lamellar doubling during annealing of polyethylene. Macromolecules. 1997;30(25):7880-7889.

- Yao Y-F, Graf R, Spiess HW, Rastogi S. Restricted segmental mobility can facilitate medium-range chain diffusion: A NMR study of morphological influence on chain dynamics of polyethylene. Macromolecules. 2008;41(7):2514-2519.

- Bai H, Deng H, Zhang Q, Wang K, Fu Q, Zhang $Z$, et al. Effect of annealing on the microstructure and mechanical properties of polypropylene with oriented shish - kebab structure. Polym Int. 2012;61(2):252-258.

- Mandelkern L, Sharma R, Jackson J. On the annealing of polyethylene crystals formed from dilute solution. Macromolecules. 1969;2(6):644647.

- Mi D, Xia C, Jin M, Wang F, Shen K, Zhang J. Quantification of the Effect of Shish-Kebab Structure on the Mechanical Properties of Polypropylene Samples by Controlling Shear Layer Thickness. Macromolecules. 2016;49(12):45714578.

- Mi D, Hou F, Zhou M, et al. Improving the mechanical and thermal properties of shish-kebab via partial melting and re-crystallization[J]. European Polymer Journal, 2018, 101: 1-11

- Li Z, Na B, Tian N, Lv R, Zou S. Enhanced molecular orientation and strain hardening in melt spun isotactic polypropylene monofilaments through partial melting recrystallization. J Appl Polym Sci. 2012;123(2):995-999. 